\title{
Entre la soberanía lingüística y la protección migratoria: la lengua como salvoconducto en la migración de Filipinas a Italia
}

\author{
Between linguistic sovereignty and protected migration: \\ language as safe-conduct in migration \\ from the Philippines to Italy \\ Maria Cama \\ Universidad de Granada \\ mariacama@correo.ugr.es \\ María Rubio Gómez \\ Universidad de Granada \\ mariarubio@ugr.es \\ Francisco Javier García Castaño \\ Universidad de Granada \\ fjgarcia@ugr.es
}

Cómo citar/Citation: Cama, Maria, María Rubio y Francisco J. García Castaño. 2020. «Entre la soberanía lingüística y la protección migratoria: la lengua como salvoconducto en la migración de Filipinas a Italia». Deusto Journal of Human Rights, No. 6: 43-68. doi: http://dx.doi.org/10.18543/djhr.1871.

Sumario: Introducción. 1. Razones de un plan para formar a migrantes filipinos italohablantes. 1.1. Las relaciones entre Italia y Filipinas. 1.2. El acuerdo de cooperación bilateral entre el Gobierno filipino y Gobierno italiano. 2. Training of trainers on Italian language and culture: una experiencia práctica. 2.1. Etapa 1: Filipinas. 2.2. Etapa 2: Italia. 3. La experiencia vista por sus protagonistas. Conclusiones. Referencias bibliográficas.

Resumen: El artículo estudia el caso de la migración filipina en Italia poniendo el acento particularmente en el uso que el país de acogida hace de determinados procesos de enseñanza-aprendizaje de la lengua propia como garantes de la integración socio-laboral de las personas migrantes procedentes del país asiático. A partir de la descripción y análisis de un 
acuerdo intergubernamental planteado por los gobiernos filipino e italiano en materia de migración y trabajo, y desde el que la adquisición de la lengua de "acogida» se traduce en mejores oportunidades migratorias y laborales, abordamos el estudio de iniciativas de este tipo en las que se evidencia el rol que en la actualidad juegan políticas migratorias que convergen en proyectos de integración orientados a modelos asimilacionistas. Finalmente, destacamos las límitaciones de una legislación que aún no reconoce explícitamente el derecho fundamental de las personas migrantes a mantener sus propios patrimonios lingüísticos y culturales.

Palabras clave: Movilidad internacional, derechos lingüísticos, formación lingüística, cooperación interinstitucional, Italia, Filipinas.

Abstract: The article presents the case of Philippine migration to Italy, with particular emphasis on the host country's use of certain teaching-learning processes of its own language as guarantors of the socio-labour integration of migrants from the South-Asian country. First, we start from the analysis and discussion of a bilateral agreement on migration and labour between the Philippine and Italian Governments that, through the acquisition of the "host» language, provides better migration and employment opportunities. Based on this, we undertake the study of such initiatives, in which migration policies implement integration projects oriented towards assimilation models. Finally, we put the emphasis on the limitations of a legislation that does not yet explicitly recognize to migrants a fundamental right: the right to maintain their own linguistic and cultural heritage.

Keywords: International mobility, linguistic rights, language training, intergovernmental cooperation, Italy, Philippines. 


\section{Introducción}

El tema del aprendizaje de las lenguas propias de los lugares de destino en contextos migratorios despierta un enorme interés por su centralidad en los discursos políticos y educativos sobre la integración de poblaciones inmigrantes (Gutiérrez et al. 2010). El hecho de dominar dichas lenguas se percibe como un factor determinante para la integración, ya que si las personas procedentes de otros países conocen los diferentes usos y funciones del lenguaje, para comprender, expresarse de forma coherente y no permanecer al margen del contexto comunicativo real (Sobrero y Miglietta 2011), sus posibilidades en el acceso a recursos, derechos, empleo, educación, etc., se multiplican. Es por ello por lo que ha aumentado la atención dirigida a la "lengua de acogida», considerada imprescindible para la comprensión interpersonal y, por consiguiente, se entiende que facilita una «integración eficaz». Las lenguas de las poblaciones migrantes, en cambio, aún no han recibido una adecuada atención en términos de recursos y reconocimiento por parte de las instituciones, cuyas iniciativas se caracterizan por una orientación hacia la asimilación (García Castaño et al. 1993). Y es que la polémica surge en torno a si las poblaciones migrantes, cuyas lenguas son distintas a la lengua mayoritaria del país de acogida, tienen derecho a usar y mantener sus conocimientos lingüísticos en espacios públicos, sobre todo en contextos educativos (May 2010).

Los fenómenos migratorios conllevan importantes cuestiones ligadas a los derechos lingüísticos de estas poblaciones, como el derecho a la protección de las minorías, el derecho a la defensa y valorización de las lenguas y las culturas de origen y el vínculo de las lenguas con la historia y la identidad de los pueblos (Biondi Dal Monte 2017). Hamel (1995a) subraya que los derechos lingüísticos (entre los cuales está el uso de su propio idioma en contextos públicos y privados) y el derecho a no sufrir discriminación debido a sus propias opciones lingüísticas, son derechos humanos fundamentales que pertenecen a todos los seres humanos, tanto a nivel individual como a nivel colectivo, es decir, a cada persona y a su comunidad. El mismo autor, a este respecto, escribe:

El hecho evidente de que un sujeto sólo pueda ejercer sus derechos individuales de comunicarse en su lengua en la medida en que exista y sobreviva su comunidad de habla demuestra que todo derecho lingüístico se basa en última instancia en la comunidad y tiene, por lo tanto, un carácter colectivo (Hamel1995a, 16). 
Desafortunadamente, dicho derecho se reconoce como natural solamente para los miembros de la comunidad lingüística dominante y las lenguas de las personas inmigrantes son difícilmente reconocidas como válidas, a nivel colectivo, en los contextos de acogida. Como evidencian algunas investigaciones en este sentido sobre contextos escolares (Broeder y Mijares 2003, Carpani, Sanfelici y Ariolfo 2011, Martín y Mijares 2007, Rubio 2015, Vila 1999) y otros estudios sobre situaciones de contacto lingüístico (Enríquez 2015, Firpo y Sanfelici 2016, Hipperdinger 2015, Pozzi 2014), para una adecuada «integración» sería necesario el reconocimiento y puesta en valor del derecho a las lenguas propias ${ }^{1}$ (las llamadas «maternas»), aspecto bastante descuidado en las políticas de gestión de la diversidad a nivel general (educativas, sociales, laborales, etc.).

En efecto, si por una parte, la creciente atención en Europa por los asuntos lingüísticos relacionados con las migraciones ha llevado al reconocimiento de gran parte de las lenguas y de las estrategias comunicativas utilizadas como capital cultural colectivo (Beacco et al. 2005), por otra, en Italia como en otros países, en nombre de una supuesta integración, se exige que las personas aprendan —o más bien dominen- la lengua de los lugares a los que llegan² (incluso en el caso que nos ocupa, antes de haber llegado) sin tener en cuenta sus lenguas de origen, subestimando el aspecto de la identificación y presionándolas para que, en lugar

1 Derechos lingüísticos vinculados a los principios de igualdad y dignidad humana, al derecho a la diversidad y en concreto a la expresión de la propia identidad (Hamel 1995b). No es asunto de este trabajo recuperar argumentos que ligan las nociones de identidad y derechos y que han sustituido, cuando no invisibilizado, en diversos foros, el debate relativo a la defensa de los derechos sociales de diversas comunidades minoritarias (Joppke 2007) entre las que se sitúan las poblaciones migrantes. Pero sí queremos dejar constancia de la importancia de dichos debates para entender en profundidad las relaciones entre ciudadanía, derechos e identidades.

2 En la evolución de las relaciones entre el idioma de acogida y las políticas migratorias de los países en los que se establecen las personas inmigrantes «con fines de residencia más o menos permanente» se centra un interesante estudio de Bermejo, que lleva a cabo un análisis comparativo de dos de los principales instrumentos de políticas públicas de diferentes países (Canadá, Australia, Nueva Zelanda, Estados Unidos, Europa) que «incorporan, abordan y configuran con diferentes fines los requisitos idiomáticos relativos a los no nacionales del país receptor» (Bermejo 2008: 2). Así describe y analiza el sistema de puntos, antes de la llegada, a fin de valorar que el conocimiento de la lengua alcance el nivel requerido para cubrir las necesidades laborales del país; y las pruebas de idioma planteadas para seleccionar a la población y que implican la obligación de alcanzar un determinado nivel de dominio lingüístico para la obtención de la ciudadanía. 
de compatibilizar los diferentes idiomas, abandonen una lengua en favor de otra (Cama 2017). Así pues, el endurecimiento de los requisitos lingüísticos exigidos a las poblaciones migrantes en diversos países de acogida ha sido, por un lado, considerado xenófobo y contrario al reconocimiento de los derechos de las personas inmigrantes (Olmos 2016, Morales 2017), y, por otro, criticado por ser discriminatorio según la línea de trabajos como los de Martín Rojo (2010), que denuncian la «descapitalización cultural» que están sufriendo poblaciones migrantes cuyas lenguas y experiencias no se están teniendo en cuenta en estos procesos de supuesta «integración».

Desde estas premisas surge el presente texto, fruto de una investigación más amplia ${ }^{3}$, llevada a cabo a través de una metodología cualitativa con el objetivo general de analizar los hábitos lingüísticos de la comunidad migrante filipina en la ciudad de Mesina (Sicilia).

En este contexto, y apoyándonos en fuentes secundarias, pretendemos centrarnos en lo relativo a una medida legislativa en materia de movilidad internacional por razones de trabajo que resulta esencial para entender los patrones migratorios que nos ocupan: el Acuerdo bilateral del 4 de noviembre de 2016 entre la República Italiana y la República de Filipinas, en el que la lengua, vista como vehículo fundamental para la integración, es el centro de una acción de cooperación internacional e interinstitucional, cuyo objetivo es facilitar la inserción laboral de las personas trabajadoras de nacionalidad filipina que migran a Italia.

Así, después de considerar las razones que llevaron a la cooperación entre Estados, se describe y analiza críticamente el Acuerdo que incluye la realización de un proyecto de formación lingüística intensiva dirigido a un grupo de personas filipinas, seleccionadas para enseñar italiano a sus conciudadanos antes de la salida del país para llegar a Italia. En la primera parte describimos las características de la inmigración filipina en Italia y las razones que llevaron a los dos países a las negociaciones y a la promoción de una acción conjunta en materia de formación y empleo. En la

3 El texto pertenece a un trabajo de tesis doctoral, en fase de finalización, que se está realizando en el Programa de Doctorado en Estudios Migratorios de la Universidad de Granada. Dicha tesis representa el resultado de un estudio cualitativo, desarrollado con jóvenes y adultos (sobre todo mujeres) de la comunidad filipina de Mesina, que abarca la compleja cuestión lingüística en contextos migratorios y aborda varios aspectos de un mismo fenómeno social: la asimilación lingüística de los individuos que se plantea en muchas ocasiones como condición indispensable para su supuesta integración en los países receptores. 
segunda parte presentamos el proyecto analizado incorporando valoraciones y análisis de parte de la experiencia formativa en Italia. Por último, concluimos con unas reflexiones sobre algunos aspectos problemáticos de las estrategias adoptadas por Italia y Filipinas en este sentido y de los mecanismos de gestión de la movilidad internacional por razones de trabajo, destacando la necesidad de un replanteamiento de las posiciones institucionales, que coartan el uso y reconocimiento de las lenguas propias de poblaciones inmigradas, hacia una política que sobrepase la limitada noción de integración vehiculada hasta el momento.

\section{Razones de un plan para formar a migrantes filipinos italohablantes}

La comunidad filipina en Italia, cuyo asentamiento empieza en los años setenta del pasado siglo, es una de las comunidades más antiguas de inmigrantes en el sur de Europa. Con 168.292 presencias registradas por el Istituto di Statistica demográfica (ISTAT 2019) esta fuente gubernamental coloca a la población de origen filipino en quinta posición en la lista de la ciudadanía no comunitaria «regularmente» residente. La primera inmigración filipina en Italia se caracterizó por estar altamente feminizada, como consecuencia de las necesidades de mano de obra en un sector (el de la asistencia doméstica) históricamente atribuido a las mujeres. Con el tiempo, se ha mantenido una mayor presencia de mujeres que, según los datos de ISTAT a 1 de enero de 2019 resultan 95.346. Aunque en el país la distribución actual entre varones (72.946) y mujeres (95.346) no es tan desigual como en el caso de la migración ucraniana (que se caracteriza por un $70 \%$ de presencia femenina), desde el principio la inmigración filipina en Italia ha estado formada en su mayoría por mujeres. Gracias a las reunificaciones familiares, durante la considerada segunda fase de la inmigración filipina a Italia, a mediados de los años ochenta se observó un relativo crecimiento de la presencia de los varones (IDOS/Immigrazione Dossier Statistico 2014). En este periodo los flujos aumentaron y se estructuraron las cadenas familiares. Gracias a las mujeres que actuaron como intermediarias, también los varones encontraron trabajo como empleados domésticos. En estos años, las personas filipinas empezaron a considerar Italia en términos de destino permanente o duradero desde el que poder mantener una actividad económica que les permitiera enviar dinero para respaldar económicamente a 
los familiares que se quedaron en Filipinas (de cara a la compra o la construcción de una vivienda, la puesta en marcha de una empresa, etc.). La segunda mitad de los años noventa se caracterizó por un mayor equilibrio entre las mujeres y los hombres que llegaron por razones de reunificación familiar (Tognetti 2004). El nacimiento y la presencia de hijas e hijos - junto con la progresiva inserción laboral, social y económica que estamos exponiendo- son hoy uno de los indicadores de la estabilización de esta comunidad (Cardullo 2010), y tanto las reunificaciones familiares como la constitución de nuevas familias son el resultado de una mejora económica y social necesaria para acoger y mantener el núcleo familiar - factores que han incrementado la cuota de permisos de larga duración del 47,4\% en 2012 al 62,2\% en 2018 (Ministero del Lavoro e delle Politiche Sociali 2018,5$)^{4}$.

Aunque no son muchos los datos oficiales que comparan la evolución, año por año, de la presencia por sexo de esta migración en Italia, existe un informe de 2010 del Dipartimento programmazione settore statistica de Bolonia (capital de una de las tres regiones de atracción de la comunidad en cuestión), que evidencia cómo ha ido cambiando la composición de la población filipina, según la variable del sexo, desde 1986 hasta 2009 en dicha ciudad, y muestra que la marcada diferencia de los primeros años entre hombres y mujeres, aunque se mantiene, poco a poco se reduce. Si, en efecto, a finales de los años ochenta, entre las 93 personas procedentes de Filipinas, las mujeres conformaban en esta ciudad más del 70\% (67 mujeres y 26 varones), a finales de los noventa la diferencia entre los dos sexos ha ido disminuyendo (1.036 mujeres y 712 hombres) hasta alcanzar un mayor equilibrio a finales de 2009, con 2.423 mujeres y 1.993 hombres (Comune di Bologna 2010).

Haciendo referencia a la distribución territorial, y siguiendo los datos de ISTAT (2019), el norte de Italia representa el primer destino para el $52 \%$ de las personas filipinas que dejan su tierra de origen para establecerse en este país. En el norte se encuentran precisamente dos de las primeras tres regiones por número de presencia filipina: Lombardía — que está en primera posición por acoger a un tercio de

4 Los informes anuales sobre la presencia en Italia de las dieciséis comunidades migrantes extranjeras numéricamente más relevantes los realiza, a partir de 2012, ANPAL Servizi, en relación con el proyecto La Mobilità Internazionale del Lavoro. La relación completa de dichos informes, ediciones 2012-2018, puede consultarse en las secciones Paesi di origine e comunità y Rapporti di ricerca sull'immigrazione del portal institucional: www.integrazionemigranti.gov.it 
esta población (34,7\%) - y Emilia-Romaña —tercera por número de personas filipinas residentes (con un porcentaje del 8,6\%)-. Una cifra considerable se registra en el centro del país, concretamente en el Lacio, región que está en segunda posición, con una concentración particular en la ciudad de Roma, donde se encuentran más de cuarenta y seis mil personas de origen filipino registradas, lo que supone el $27,6 \%$ del total. Así, reside en las regiones del centro el 38,5\% de la comunidad en cuestión, con importantes presencias también en Toscana, que acoge el 7,9\% del total de la población filipina en Italia (ISTAT 2019).

En cuanto al sur de Italia, Sicilia es la región meridional donde se encuentra el porcentaje más alto (3,2\%), seguida por Campania $(2,3 \%)$ y Calabria $(1,7 \%)$. Centrándonos en la primera ubicación, la comunidad filipina fue una de las primeras comunidades de origen no europeo en llegar a Sicilia; tierra que, hace algunas décadas, pasó de ser zona de tránsito de millares de personas procedentes de otros países a lugar definitivo de residencia para muchas de ellas. Entre las provincias sicilianas, Mesina se ha mostrado la más atractiva para las personas procedentes de Filipinas, gracias a la demanda creciente en el sector doméstico y asistencial, a la creación de puentes ligados al factor religioso y por el Philippine Overseas Labour Office (POLO) ${ }^{5}$. Entre las personas procedentes del continente asiático, la comunidad filipina (19,2\%) ocupa en Mesina el segundo lugar después de la comunidad originaria de Sri Lanka $(32,4 \%)$ y el cuarto lugar entre las personas originarias de los cinco continentes. En toda su provincia las personas de origen filipino son 2.494 , con un número mayor de mujeres con respecto a los varones (1.162 varones y 1.332 mujeres). Casi la mitad de las 5.317 personas filipinas (2.292 varones y 3.025 mujeres) instaladas en Sicilia, viven y trabajan en Mesina, 1.817 en Palermo, 600 en Catania, 170 en Siracusa, 101 en Enna, 59 en Agrigento, 49 en Ragusa, 23 en Trapani y 4 en Caltanissetta (ISTAT 2019).

Si hasta finales de los años ochenta este tipo de inmigración estuvo bastante circunscrita, a principio de los noventa se convirtió en un fenómeno más consistente que se consolidó con la presencia de una extensa red parental/amistosa capaz de guiar los flujos de familiares y conciudadanos (Banfi 2008, Malfone 2006, Favaro y Tognetti 1991). La inserción de las mujeres filipinas, principalmente en

5 El Philippine Overseas Labour Office es un organismo que actúa como agente del Department of Labor and Employment (DOLE) para la aplicación de sus políticas y programas hacia las personas trabajadoras en el extranjero. 
el sector doméstico y de asistencia a las personas, responde, desde el principio, a la demanda del mercado laboral italiano, determinada por factores demográficos y socioeconómicos que obligan a las familias a recurrir a formas de asistencia externa de naturaleza privada. Debido, entre otros factores, al envejecimiento de la población autóctona, la pérdida de la familia extensa, la falta de adecuados servicios públicos de atención a las familias, la emancipación de las mujeres con la disminución de la posibilidad de hacerse cargo de las personas mayores y de las niñas y niños y las consiguientes menores limitaciones económicas en el seno de los núcleos familiares, se crea en el mercado italiano una oferta de mano de obra en el sector de los servicios sociales y de asistencia que constituye un importante factor de atracción de la inmigración filipina la cual, como ya hemos explicitado, se convierte con el tiempo en una inmigración de tipo familiar - prueba de ello es que a partir de 2010 el mayor número de permisos de residencia se expiden por razones de reunificación familiar (Ministero del Lavoro e delle Politiche Sociali 2018)—. Desafortunadamente, la movilidad de las personas filipinas hacia otros ámbitos laborales es difícil6. A pesar del alto nivel de educación conseguido en el país de origen por los miembros de la comunidad filipina en Mesina (el $51 \%$ posee una titulación de segundo grado y el $12 \%$ un nivel de instrucción universitario), su inserción laboral en otros campos se ve fuertemente obstaculizada por la falta de reconocimiento de sus titulaciones y por el escaso conocimiento de la lengua italiana, cuyo aprendizaje, casi siempre espontáneo, está condicionado por el tipo de trabajo que desempeñan. Así, las estadísticas muestran que ocupan exclusivamente posiciones laborales no cualificadas: colaboradores domésticos, personal de servicio, personal de limpieza de oficinas, tiendas, restaurantes, hoteles, depósitos, embarcaciones (Ministero del Lavoro e delle Politiche Sociali 2018) ${ }^{7}$. Aun así, en la mentalidad de la población autóctona, ser de Filipinas es ser considerada como una persona «perfecta como colaboradora doméstica», atribuyendo a esta población características

6 En realidad, la limitada movilidad laboral ascendente no afecta exclusivamente a la comunidad filipina. De hecho, Fellini, Guetto y Reineri (2018) detectan las problemáticas relacionadas en este sentido entre las personas inmigrantes en Italia procedentes de países no occidentales: personas que, a pesar de tener niveles de educación superior a la secundaria obligatoria en el país de origen, ocupan en el mercado laboral de los países de «acogida» nichos laborales para los que no se precisa cualificación.

7 Datos elaborados por la Dirección de transición de grupos vulnerables - ANPAL Servizi en datos del Ministero del Lavoro e delle Politiche Sociali - Sistema informativo de las Comunicaciones Obligatorias. 
muy bien valoradas por la población italiana en este sentido, como la fiabilidad o la laboriosidad (Basa y De la Rosa 2004).

$\mathrm{Si}$, por un lado, la especialización profesional ha mantenido a esta población en una posición de subordinación y "segregación» ocupacional, por otro, como destaca también el último análisis publicado por el Ministero del Lavoro e delle Politiche Sociali (2018), la ha protegido de las graves repercusiones de la crisis que ha caracterizado la economía italiana en los últimos años. De este modo, cabe destacar que, entre las poblaciones migrantes no comunitarias, la comunidad filipina mantiene la tasa de empleo más alta (79,3\%), y concretamente entre las mujeres filipinas se encuentra la tasa más baja de desempleo $(5,3 \%)$ e inactividad $(17,7 \%)$ con respecto a los valores medios de las personas no comunitarias, que alcanzan un $18 \%$ y un $44 \%$ respectivamente.

\subsection{Las relaciones entre Italia y Filipinas}

Con estos datos de movilidad y empleo, se explica que el Gobierno de Filipinas haya promocionado la emigración desde los años setenta, cuando empezó a adoptar una política dirigida a contrarrestar el masivo desempleo local y a incrementar la economía del país gracias a las remesas (Bea 2012). Para comprender el alcance de este fenómeno, debemos tener en cuenta la falta de despegue económico del país, la inestabilidad política, las catástrofes naturales y todo ello sumado a uno de los crecimientos demográficos más elevados del mundo. Por estas razones, los distintos Gobiernos filipinos, a través de agencias gubernamentales dependientes del Ministerio del Trabajo, han fomentado la emigración y, en el imaginario colectivo, la persona que emigra se ha representado como un héroe y/o heroína nacional (bayano en tagalo). La exportación de mano de obra se ha convertido con el tiempo en una acción central de las políticas públicas, hasta el punto de que se creó una agencia gubernamental, la Philippine Oversaes Employment Administration (POEA), con la misión de facilitar el acceso al empleo de las personas migrantes en el exterior. En Europa, Italia es la segunda fuente de remesas para la población filipina inmediatamente después del Reino Unido. Estas personas trabajadoras inmigrantes son a menudo la principal fuente de ingresos para la familia y la comunidad en general en Filipinas. El de las remesas sigue siendo un mercado en continua expansión, con ocho personas inmigrantes de cada diez que envían sus ahorros al 
país de origen y una multiplicación evidente de agencias de money transfer (Colaiacomo 2012).

Por la amplitud de los flujos migratorios de Filipinas hacia Italia y «para asegurar una gestión más eficaz de los grandes flujos migratorios entre los dos países» ${ }^{8}$ (TESDA 2015, 2) ${ }^{9}$, las relaciones bilaterales entre el Gobierno italiano y el Gobierno filipino, que se basan desde el 17 de junio de 1988 en una estrecha cooperación cultural, culminaron en un Acuerdo bilateral en materia de movilidad internacional.

Siguiendo diferentes etapas, las negociaciones iniciadas en 2012 dieron lugar a la concreción del texto del Acuerdo que nos ocupa durante una reunión celebrada en Manila (27 de junio de 2014) entre los representantes del Ministero del Lavoro e delle Politiche Sociali y del Department of Labor and Employment (DOLE). Dicho texto incluye la regulación de diversos aspectos como reforzar la cooperación en la gestión de las migraciones por razones de trabajo, mejorar las modalidades de selección de mano de obra para responder efectivamente a las «exigencias» del mercado laboral italiano, o aportar e intercambiar herramientas técnicas (fichas de datos, listados de personas trabajadoras, estándares de formación, etc.) para una programación compartida de los flujos migratorios hacia Italia.

\subsection{El Acuerdo de cooperación bilateral entre el gobierno filipino y gobierno italiano}

El Acuerdo de Cooperación entre Italia y Filipinas, cuyo texto oficial está publicado en la Gaceta Oficial italiana n. ${ }^{\circ} 54$ del 14 de noviembre de 2017, forma parte de una serie de acuerdos bilaterales ${ }^{10}$ celebrados por la administración italiana para la reglamentación de los flujos migratorios en cooperación con las autoridades competentes de los países de origen de las personas

8 Traducción propia.

9 TESDA, Technical Education and Skills Development Authority (Filipino: Pangasiwaan sa Edukasyong Teknikal at Pagpapaunlad ng Kasanayan), es una agencia gubernamental filipina que actúa como organismo competente en materia de formación profesional y de enseñanza superior. En calidad de agencia gubernamental, está encargada de gestionar y supervisar la enseñanza técnica y profesional dirigida a los trabajadores filipinos, proporcionando políticas y actuando programas de calidad, con el objetivo de desarrollar capacidades y habilidades que puedan facilitar la inserción laboral.

10 Italia ha firmado acuerdos bilaterales también con Marruecos (2005), Egipto (2005), Albania (2008), Moldavia (2011), Sri Lanka (2011) y Mauricio (2012). 
trabajadoras inmigrantes. Los objetivos son diversos y van desde favorecer el intercambio recíproco de informaciones sobre las necesidades deducibles del mercado de trabajo italiano y de las personas profesionales disponibles en el país de origen, a elaborar y regular listados de personas interesadas en trabajar en Italia, 0 poner en marcha iniciativas de formación profesional vinculadas a las necesidades del mercado laboral italiano. Así, la puesta en marcha de programas de aprendizaje de "lengua y cultura italiana» antes de la salida del país, se hace imprescindible para la adquisición de derechos de prelación de cara a la entrada a Italia ${ }^{11}$.

El citado Acuerdo prevé, entre otras cuestiones, que ambas partes faciliten la formación lingüística y profesional de las personas que, desde Filipinas, pretenden trabajar en Italia. En este sentido, se especifica que el Gobierno italiano costeará los cursos de formación en Filipinas - dentro de los límites de los recursos humanos, materiales y económicos establecidos por la legislación nacional italiana vigentey que las personas que hayan finalizado con éxito dichos cursos de formación lingüística tendrán prioridad para la entrada en Italia (Capítulo IV, art. 12, apartados 1, 2 y art. 13). Es decir que, aunque para iniciar un proyecto migratorio no es obligatorio que cumplan la formación antes de salir, quienes asisten a estos cursos adquieren derechos de preferencia, una especie de salvoconducto a Italia (art. 13). Por su parte, el Gobierno filipino facilita los listados de personas que han completado la formación de donde las empresas italianas y las entidades autorizadas podrán seleccionar los candidatos (art. 8). Todo ello con una clara orientación de gestión de flujos migratorios entre ambos países.

Respaldando las negociaciones entre los dos países, se ha diseñado y organizado un plan orientado a la formación de personas formadoras financiado en su totalidad por el Gobierno italiano y dirigido a docentes en Filipinas. Se pretende que estos docentes puedan impartir cursos de 150 horas de «lengua y cultura italiana» de nivel A2 en las áreas de mayor emigración hacia Italia (como Batangas, al sur de Manila), de los que podrán beneficiarse las

11 A efectos de la aplicación del art. 23 del Decreto Legislativo n. 286 del 25 de julio de 1998, Ilamado Testo Unico delle disposizioni concernenti la disciplina dell'immigrazione e norme sulla condizione dello straniero. El Texto Único es un articulado conjunto de normas que regula la inmigración en Italia, que se compone de 49 artículos. En el art. 23 se prevén actividades de educación y formación profesional en los países de origen que tendrían como finalidad la incorporación en el sistema laboral italiano y darían facilidades para aquellas personas que hayan asistido a estas actividades. 
personas que quieren establecerse en Italia por razones de trabajo o de reunificación familiar.

\section{Training of trainers on Italian language and culture: una experiencia práctica}

El plan titulado Training of trainers on Italian language and culture, elaborado en Manila por el National Language Skills Institute de la Technical Education and Skills Development Authority (TESDA), en el marco del proyecto La Mobilità Internazionale del Lavoro - que como indica su título, tiene el objetivo de formar a docentes filipinos expertos en «lengua y cultura italiana»-, se inserta en una intervención previamente diseñada con una finalidad más amplia (favorecer la movilidad por razones de trabajo) y que, por lo que respecta a Italia, es competencia del Ministero del Lavoro e delle Politiche Sociali. Este se vale de la colaboración de Italia Lavoro Spa (Anpal servizi Spa desde el 1 de enero de 2017) y de organismos de formación acreditados, que tienen la tarea de proporcionar materiales, supervisar los resultados de las personas participantes, evaluar el dominio del idioma y presentar informes sobre el logro de dichos/as profesionales. En cuanto a la parte filipina, la autoridad competente es el Department of Labor and Employment (DOLE) que valiéndose del apoyo de sus agencias gubernamentales: la Philippine Overseas Employment Administration (POEA), el Philippine Overseas Labour Office (POLO) y el Overseas Workers Welfare Office (OWWO) se ocupa de asistir a las personas filipinas que trabajan en el exterior y asegurar el respeto de sus derechos fundamentales. Por otro lado, encontramos la Technical Education and Skill Development Authority (TESDA) que, en este caso, es responsable de reclutar desde las principales áreas filipinas de emigración a las personas participantes en las actividades de formación, proporcionar la primera parte de la formación en Filipinas y ocuparse de la asistencia administrativa, de la supervisión y de la evaluación de la formación (monitorización y evaluación). Y, por último, la Professional Regulation Commission (PRC), cuya tarea es supervisar el funcionamiento general del programa.

El plan concreto de formación para docentes que describimos en este trabajo se organizó en dos fases distintas: la llamada «Etapa 1», desarrollada en Filipinas entre noviembre y diciembre de 2015 y la «Etapa 2», llevada a cabo en Italia y terminada en noviembre de 2016. 


\subsection{Etapa 1: Filipinas}

La experiencia filipina se desarrolló en Manila, en las aulas del National Language Skills Institute de TESDA, y se articuló en dos periodos de formación lingüística, denominados Fase I y Fase II. Las personas participantes, seleccionadas por TESDA, tenían que cumplir determinados requisitos: poseer un título universitario de tres años, tener una edad inferior a 45 años y al menos un año de experiencia previa en la enseñanza de una lengua extranjera, y, además de completar con éxito todas las fases del programa de formación, comprometerse a trabajar con TESDA durante un periodo mínimo de tres años. La Fase I consistió en un primer curso intensivo de lengua de 300 horas organizadas en un mínimo de 30 horas semanales durante seis días por semana. Las actividades formativas se realizaron, utilizando medios de enseñanza y aprendizaje interactivos, a través de material didáctico e instrumentos técnicos audiovisuales. Las clases fueron impartidas por docentes italianos nativos con al menos dos años de experiencia y con la presencia en el aula de dos tutores. Los estudiantes tuvieron que asistir al $70 \%$ de las clases para ser admitidos a un primer examen destinado a verificar su adquisición del nivel B1 del Marco Común Europeo. Una vez aprobado el examen fue posible pasar a la llamada Fase II, la segunda parte de la formación en el país, cuyo objetivo era ofrecer a sus participantes la oportunidad de lograr una certificación de nivel B2 del Marco Común Europeo por uno de los entes acreditados y reconocidos por el Ministero degli Affari esteri e della Cooperazione Internazionale italiano. Esta parte consistió en un curso intensivo de 150 horas de clases de lengua y metodología, desarrolladas (como en la Fase I) con técnicas de aprendizaje interactivo por docentes con lengua materna italiana, con vistas a mejorar las capacidades de comprensión oral, comprensión escrita, producción escrita y producción oral de cara a adquirir las habilidades necesarias para enseñar italiano a personas filipinas que quisieran establecerse en Italia por razones familiares o de trabajo. El pago de la inscripción al examen, así como todos los gastos relativos a la expedición de las certificaciones y los costes de los libros de texto, del material educativo, del desplazamiento, del alojamiento y de la cobertura de seguro, hasta la conclusión de cada fase de las actividades, correspondieron al Estado italiano (más bien a la empresa adjudicataria de una licitación pública12).

12 Fue la International Language School Srl que, tras una licitación pública, se adjudicó el contrato para proporcionar el servicio. 
Al mismo tiempo, se aseguró un servicio de tutoría y la presencia de al menos un responsable de la coordinación de las acciones formativas. Además, el servicio incluía una beca diaria para cada estudiante de 700 pesos filipinos (correspondientes aproximadamente a 12 euros) para sufragar los costes de las comidas. Los estudiantes que superaron esta primera parte del entrenamiento se incluyeron en la Etapa 2, el segundo paso formativo que tuvo lugar en Italia, llamado Rafforzamento in bagno linguistico delle competenze linguistiche, culturali e didattiche di 12 docenti di lingua italiana della Tesda- Repubblica delle Filippine ${ }^{13}$.

\subsection{Etapa 2: Italia}

La etapa italiana, constituida por una fase de inmersión lingüística en Roma, estaba dirigida a mejorar las competencias lingüísticas, metodológicas y culturales adquiridas en la Etapa 1 para que las personas participantes pudieran ser contratadas por TESDA como formadoras lingüísticas para sus conciudadanos/as en Filipinas. Aunque no se explicitaron las razones en las que se basó la decisión de elegir como lugar Roma, podemos suponer que dicha elección se debió a razones de tipo práctico, considerando que en esta ciudad se encuentran las sedes del Gobierno italiano y de la Embajada Filipina en Italia y que aquí reside una numerosa comunidad filipina con larga historia de inmigración compartida. Las personas estudiantes que participaron en la formación impartida en Roma realizaron un curso de 155 horas (135 de actividades realizadas en clase y 20 de excursiones a diversos lugares), gestionado por ItaliaLavoro Spa y la International Language School Srl, con el —oficialmente- doble objetivo de ampliar las oportunidades de aprendizaje de la lengua y de conocer el contexto social del país de destino para poder adaptar los programas de enseñanza de lengua y cultura italiana previos a la salida del país «a las exigencias reales de las personas incluyendo contenidos indispensables para poder superar las dificultades de una primera inserción laboral y social en Italia» (Ministero del Lavoro e delle Politiche Sociali 2016, 3). Con el fin de completar el conocimiento sobre el contexto italiano, el servicio estableció una alternancia de lecciones de lengua y glotodidáctica en el aula con visitas guiadas a entidades e instituciones competentes en materia de

13 «Fortalecimiento de las competencias lingüísticas, culturales y didácticas de 12 docentes de lengua italiana de Tesda- Republica de Filipinas mediante curso de inmersión lingüística» (Traducción propia). 
migraciones e integración ${ }^{14}$. Dichas visitas se complementaron con seminarios sobre la normativa italiana en materia de migración y trabajo y sobre herramientas de orientación, como el Portale integrazione migranti ${ }^{15}$, para poder facilitar todo tipo de información útil «para el éxito de los procesos de integración de las personas extranjeras en la primera entrada» y «una progresiva y rápida capacitación de sus conciudadanos ya incluidos en el país de destino» (ItaliaLavoro 2015, 1). Al final, cada uno de los participantes tuvo que hacer dos pruebas sobre competencias metodológicas y socioculturales y llevar a cabo un Project Work individual, dirigido a las personas conciudadanas que quieren emigrar a Italia. En lo relativo a las competencias socioculturales, cabe destacar que se hacía expresamente referencia al conocimiento de cuatro ámbitos distintos:

1. Entrada a Italia, Permisos de residencia, instituciones y modalidades de acceso a los servicios públicos (Sportello Unico per I'Immigrazion ${ }^{16}$, Ayuntamiento, Registro, servicios socio-sanitarios, servicios educativos, Patronatos, INPS $\left.{ }^{17}\right)$. 2. Trabajo en Italia y formación profesional (centros de empleo, centros de formación profesional, CPIA, centros de educación para adultos, trabajo autónomo). 3. Vida cotidiana (transportes, comercios, lugares de culto, espacios públicos, etc.). 4. Características de la comunidad filipina en Italia (ItaliaLavoro 2015, 3) ${ }^{18}$.

14 La formación contaba con 155 horas en total, 135 de las cuales se desarrollaban en el aula, distribuidas en 90 horas dirigidas a fortalecer las habilidades lingüísticas con vistas a obtener la certificación $\mathrm{C} 1$; 35 horas destinadas a mejorar la capacidad de diseño de programas de lengua y cultura italiana previos a la salida del país y a su adaptación a las necesidades de los usuarios y10 horas centradas en la preparación y la consiguiente valoración de las visitas guiadas. Para las restantes 20 horas se planificaron visitas en el territorio en un máximo de cuatro jornadas de cinco horas. El acceso a estas informaciones fue posible gracias a la colaboración de la Project manager responsable de coordinar el proyecto que nos envió explicaciones al respecto, actas y un documento multicopiado en el que se describe en detalle el servicio proporcionado por Italia Lavoro SpA (hoy ANPAL servizi SpA).

15 El Portal tiene el objetivo de facilitar el acceso a los servicios para la integración, asegurando a toda la población una correcta información.

16 La Ventanilla Única para la Inmigración se encuentra en cada Prefectura y se ocupa de expedir, tras haber verificado los requisitos, las autorizaciones necesarias para las contrataciones de las personas extranjeras no comunitarias y los permisos de residencia por razones de reunificación familiar.

17 El Istituto Nazionale della Previdenza Sociale (INPS) es la principal entidad del servicio público italiano de pensiones en el que deben obligatoriamente inscribirse todas las personas trabajadoras empleadas públicas y privadas y la mayor parte de las personas trabajadoras autónomas. Se encuentra bajo la supervisión del Ministero del Lavoro e delle Politiche Sociali.

18 Traducción propia original del italiano. 
Durante todo el periodo de su estancia en Roma a las personas participantes se les garantizó la asistencia necesaria en términos de recursos económicos (desplazamientos, comida, alojamiento, cobertura de seguro, material didáctico) y humanos, es decir, docentes con al menos tres años de experiencia en enseñanza de lengua italiana a personas extranjeras, presencia de al menos un tutor en clase y de un mediador cultural durante las visitas guiadas en el territorio. En este sentido, cabe destacar que fue una iniciativa única, un esfuerzo sin precedentes desde el punto de vista de la participación tanto práctica como económica del Gobierno italiano.

Las actividades se concluyeron con la presentación de los objetivos de la formación lingüística previa a la salida del país a un grupo de representantes de la comunidad filipina residente en Italia durante una reunión dedicada al papel de esta comunidad en Italia, en colaboración con la Embajada de Filipinas. En dicha reunión, que se realizó el 14 de noviembre de 2016 en la sede de Italia Lavoro Spa, participaron todos los representantes de las instituciones involucradas de los dos países: la directora general de inmigración del Ministero del Lavoro e delle Politiche Sociali italiano, los representantes de la International Language School -adjudicataria del contrato de formación-, el cónsul general de Filipinas, el Executive Director Qualifications and Standards Office de la agencia TESDA y el Labour attaché del Philippine Overseas Labour Office (POLO). Participaron también, como representantes de Italia Lavoro, el coordinador del área de inmigración y la gestora de proyectos (project manager). El compromiso con la formación para la integración de la población migrante filipina en Italia parecía evidente ante el apoyo institucional directo que dicho programa recibió.

\section{La experiencia vista por sus protagonistas}

Cuando tuvimos noticias de esta medida, la formación acababa de concluirse en todas sus fases, incluida la experiencia italiana. Por ello, no pudimos interactuar personalmente con las personas filipinas que se beneficiaron de ella, que ya habían regresado a su país para empezar su experiencia como formadoras de «lengua y cultura italiana» ${ }^{19}$. Pero,

19 En este sentido queremos destacar que el hecho de que ésta haya sido la primera experiencia de formación así organizada nos ha impedido interactuar directamente con personas migrantes que se hayan beneficiado de dicha formación en origen para migrar a Italia y poder interpretar y valorar la repercusión de dicha medida en las nuevas personas migrantes filipinas en Italia. 
indagando sobre dicho evento, logramos relacionarnos con Anpal Servizi Spa (antes de 2017 Italia Lavoro), en particular con la Project Manager, la directora responsable de gestionar este proyecto, quien nos facilitó datos relevantes, opiniones centrales y documentos básicos para poder entender el programa desde un punto de vista que iba más allá de la mera normativa. Ella misma acogió con agrado nuestro «interés y atención» por las medidas de Anpal Servizi, en particular en lo relativo a la formación de lengua italiana que nos ocupa, precisando que esta actividad de formación fue:

Una medida de apoyo al Acuerdo entre Italia y Filipinas sobre la gestión conjunta de la migración por razones de trabajo que Anpal Servizi, en calidad de agencia técnica del Ministerio del Trabajo y titular de los recursos económicos del proyecto La Mobilità Internazionale del lavoro, ha proyectado, organizado y financiado, y que se inserta en una intervención planificada más amplia, financiada enteramente por recursos italianos, para apoyar los acuerdos y las negociaciones que la Dirección General para la inmigración entabla con los terceros países para la gestión de los flujos migratorios (Project Manager Anpal Servizi Spa) ${ }^{20}$

\section{La directora nos comentó que el proyecto la Mobilità Internazionale del Lavoro:}

Tiene una línea específica de desarrollo de las relaciones institucionales con las Misiones Diplomáticas, los Ministerios análogos y con los sujetos técnicos y las comunidades extranjeras presentes en Italia. En este caso, el objetivo del Gobierno italiano fue respaldar a la TESDA a la hora de estructurar y desarrollar cursos de italiano previos a la salida del país, dirigidos a ciudadanos filipinos que habían presentado una solicitud de visado para entrar a Italia (Project Manager Anpal Servizi Spa).

Los docentes seleccionados para la inmersión lingüística en Roma resultaron, en efecto, doce: siete mujeres y cinco varones (menores de 45 años y con dos años de experiencia docente en lengua extranjera, como ya anunciamos al detallar los requisitos del programa), originarios de distintas zonas de Filipinas (Batangas, Laguna, Oriental Mindoro,

20 Todos los discursos que se presentan a partir de ahora son originales en italiano, las traducciones son propias. La entrevista a la Project Manager Anpal Servizi Spa forma parte del trabajo de campo propio, las entrevistas a los profesionales filipinos fueron realizadas y distribuidas por la institución organizadora de los cursos. 
Cavite, Bulacan, Rizal, Tarlac City, Quezon City y Taguig City). Aunque al final no todos lograron plenamente el nivel de competencia exigido, la valoración general del programa fue muy positiva, tal como se desprende de sus declaraciones personales disponibles en la web en el Portale Integrazione Migranti21. Un docente originario de Batangas, por ejemplo, contaba cómo se enteró del proyecto de formación, logrando ser incluido en el mismo en un periodo de su vida difícil en lo relativo al ámbito laboral:

En un periodo durante el cual no tenía trabajo, haciendo una búsqueda en el sitio web de TESDA, noté que estaban contratando a docentes de lengua. Me puse en contacto con ellos y me mandaron a la Oficina Regional de Batangas. El director me propuso ir a la sede central de TESDA, en Manila, donde empezó mi participación en este proyecto. Para mí es una importante oportunidad: podremos ser profesores de italiano para nuestros conciudadanos y ayudarlos a reunirse con sus familiares, a vivir y a trabajar en Italia. La lengua italiana es difícil, un verdadero desafío. Participar en este proyecto es motivador y apasionante (Christian Montealto de Batangas).

También aquellos profesionales que fueron elegidos directamente por sus centros de trabajo, para los que la decisión inicial de participar en el programa no había sido propia, valoraron positivamente la experiencia de formación, como en el caso de una de las docentes que comentaba:

La escuela donde daba clase de inglés me eligió para participar en este proyecto. Para mí es una experiencia muy útil, porque estoy aprendiendo muchas cosas, estoy enriqueciendo mi experiencia profesional en el ámbito educativo y podré trasmitir mis conocimientos a nuevos estudiantes en Filipinas. Esta experiencia es interesante y estimulante y, con ánimo y paciencia, todos lo haremos bien (Mary Jomen Dayrit, de Tarlac City).

Otra mujer participante dijo que estaba enseñando inglés a personas extranjeras en Filipinas cuando la responsable de la escuela

21 El Portale Integrazione Migranti nació en 2012 bajo la coordinación de la Direzione Generale dell'Immigrazione e delle Politiche di Integrazione del Ministero del Lavoro e delle Politiche Sociali. Las entrevistas a los profesionales filipinos se registraron el 14 de noviembre de 2016 y se recuperaron para su análisis el 10 de diciembre de 2017 de la web: http://www.integrazionemigranti.gov.it/Attualita/Notizie/Pagine/ Saremo-docenti-di-italiano-nel-nostro-Paese-e-potremo-preparare-le-persone-chevogliono-ricongiungersi-ai-propri-cari.aspx. 
de TESDA, en la que ya trabajaba, la eligió como posible candidata para participar en el proyecto, lo que supuso para ella una importante ocasión de cara a mejorar sus competencias profesionales, al mismo tiempo que podía ayudar a otras personas:

Antes de salir estaba muy entusiasmada. La experiencia que estoy viviendo es apasionante y divertida. Estoy muy agradecida por haber tenido esta oportunidad, porque es útil para mí y también lo será para otras personas. He podido aprender muchas estrategias y técnicas de glotodidáctica que podré utilizar cuando enseñe italiano en Filipinas. Vivir aquí en Italia me ha ofrecido la posibilidad de aprender muchísimas cosas también sobre la cultura italiana que trasmitiré a mis estudiantes (Flora Grace B. Ángeles, de Tarlac City).

Algo similar comentaba otro participante que fue contactado directamente por TESDA:

Yo ya estaba trabajando como docente de inglés e informática, cuando TESDA me seleccionó para participar en este proyecto gracias a mi formación en el ámbito lingüístico. La experiencia que estoy viviendo es magnífica. Roma es la mejor ciudad del mundo y los profesores están muy preparados. La lengua es el instrumento de comunicación y participación más importante y conocer varias lenguas significa poder ser un eficaz comunicador. Además, en mi caso podré utilizar mis competencias para una adecuada preparación de las personas que quieren estudiar, trabajar o reunirse con su familia en Italia (Renan Gonzales de Laguna).

Las declaraciones de las personas entrevistadas nos muestran la visión «positiva» y la satisfacción de los profesionales filipinos, que parecen orgullosos de poder formar parte de un proyecto que pretende "ayudar» a conciudadanos que quieren trabajar o reunirse con sus familias en Italia, y resaltan las bondades de un programa de capacitación que, desde nuestro punto de vista, no pretende sino controlar los flujos migratorios situando el dominio de la lengua italiana como requisito fundamental para la supuesta integración de la población filipina. Igualmente, desde la entidad organizadora, también se nos muestra satisfacción hacia lo que consideran el buen éxito de un programa que, como se deduce de los certificados y los documentos que hemos podido consultar a través de la responsable del proyecto, ha sido planeado detalladamente, en cuanto a organización, financiación y formación con el objetivo de mejorar la movilidad internacional por razones de empleo. 
Pero con los datos con los que contamos, podríamos también interpretar que detrás de este tipo de programas se esconde la idea de que estos flujos migratorios inevitables (por las duras condiciones económicas del país de origen) necesitan ser controlados, moldeados $y$, en parte, asimilados por el país de destino. Así, el gobierno filipino, lejos de fortalecerse para retener a su ciudadanía, posibilita, impulsa y apoya medidas para habilitarles para la migración, reconociendo a la lengua de acogida una función «facilitadora» para la inserción laboral y social. Al mismo tiempo, el gobierno italiano financia estos programas con la intención de gestionar la migración filipina cuya percepción social se construye desde la idea de la «minoría modelo», es decir, desde la lógica de considerarse como una población minoritaria bien adaptada, que pasa desapercibida, de la que se tiene una imagen social positiva (Basa y De la Rosa 2004) y que no supone una amenaza para la población autóctona dado el encasillamiento social y laboral en el que se les ubica.

\section{Conclusiones}

Tras conocer en profundidad el plan que hemos descrito, nos habría gustado haber podido integrar en el análisis del mismo el punto de vista de los docentes que se vieron inmersos en esta iniciativa. ¿Realmente lograron, con 20 horas de visitas programadas en Roma conocer "la cultura italiana»? ¿Qué imagen se llevaron de esta realidad? ¿Es la que habitan la mayoría de la población migrante filipina en Italia? ¿Qué lugar ocupa la lengua propia en todo este despliegue de herramientas para «facilitar la integración»? Son cuestiones en las que pretendemos avanzar en investigaciones futuras, pero desde los datos con los que ahora contamos podemos subrayar algunos aspectos que consideramos significativos.

Sin duda, la cuestión del aprendizaje de la lengua de acogida llega a ser considerada en los discursos gubernamentales tan importante para la «integración» de las personas migrantes que los Gobiernos se comprometen hasta el punto de realizar acuerdos para que estas personas trabajadoras lleguen al país de destino ya con formación en términos de competencias lingüísticas. Lo que se busca en esa mencionada «integración» es contar con trabajadores y trabajadoras que no tengan - ni causen- problemas por el desconocimiento de la lengua en los centros de trabajo y, en el caso específico del Acuerdo entre Italia y Filipinas, Italia no solo se compromete económicamente para obtener mano de obra que sepa 
hablar la lengua a su llegada sino también da prioridad y asegura el derecho a la migración a las personas que hayan participado en cursos de formación antes de salir. Por supuesto, las acciones conjuntas para la formación lingüística y profesional (aunque resultantes de fuertes intereses económicos y de control de los flujos migratorios) pueden considerarse una medida que garantiza, al menos en teoría, unos derechos humanos básicos a muchas personas que deciden emprender un proyecto migratorio. Los pasos dados, sin embargo, no nos parecen suficientes y, si tenemos que referirnos a los resultados esperados, opinamos que, pese al trabajo hasta el momento realizado, el modelo de integración que los Gobiernos persiguen se asemeja más a la asimilación (García Castaño et al. 1993) que a una verdadera medida de integración que suponga el reconocimiento de las personas migrantes y sus circunstancias.

Como hemos mostrado, se han puesto en marcha iniciativas relevantes de cara a que estas personas migrantes adquieran la lengua de acogida, pero no se han tomado medidas — al menos que estén documentadas - basadas en "aprovechar» las competencias lingüísticas, culturales y educativas que estas ya poseen. Entre otras cuestiones relevantes, como ya hemos apuntado, esta falta de reconocimiento estaría, por ejemplo, impidiendo la movilidad laboral ascendente hacia profesiones más cualificadas (Fellini et al. 2018) e infravalorando el capital humano que esta población posee y puede aportar a la sociedad de acogida. Promover planes gubernamentales en este sentido, no solo sería un justo reconocimiento de los derechos de estas personas, sino que podría ser un importante recurso, en términos tanto culturales como económicos, para las mismas sociedades a las que llegan, en las cuales la diversidad se está interpretando como diferencia que genera desigualdad (García Castaño et al. 2018).

Según estos principios, consideramos necesario un replanteamiento de las posiciones de partida hacia una política integral que permita a las sociedades pensar de manera diferente y actuar apoyando verdaderos procesos de inclusión que se basen en el reconocimiento de los propios capitales lingüísticos y culturales de las personas que se establecen en ellas ${ }^{22}$. Solamente así se podría contribuir a hacer efectiva la lucha contra las discriminaciones y la promoción de iguales

22 Consideramos que se hace imprescindible el reconocimiento de los derechos lingüísticos, orientados, como apuntaba Kloss (1977), tanto a la tolerancia (desde el respeto del uso de las lenguas en ámbitos privados y sociales) como a la promoción (desde un reconocimiento, promoción y puesta en valor a nivel gubernamental de dichos capitales lingüísticos). 
oportunidades entre las personas inmigrantes de diversos orígenes y las poblaciones autóctonas (aunque tenemos serias dudas de que este sea el deseo de los gobiernos de los países de acogida). Sin duda, las iniciativas llevadas a cabo demuestran que con voluntad política la cooperación es posible, si bien ahora deberíamos ver si esta podría materializarse en otros aspectos importantes como el reconocimiento de los títulos académicos, las competencias profesionales y por supuesto la valoración de las lenguas de origen.

Visibilizar las necesidades de la población migrante a través de iniciativas que le aseguren, entre otros, también el derecho a ver reconocido su patrimonio lingüístico y cultural de origen, es un reto urgente para una sociedad que quiera ser realmente inclusiva y que pretenda garantizar a toda su ciudadanía el reconocimiento de los derechos humanos fundamentales.

\section{Referencias bibliográficas}

Banfi, Ludovica. 2008. «Lavoro domestico, politiche migratorie e immigrazione filippina. Un confronto tra Canada e Italia». POLIS XXII, n. 1: 5-34.

Basa, Charito y Rosalud De la Rosa. 2004. Io, noi e loro: realtà ed illusioni delle collaboratrici familiari filippine. Un progetto di ricerca comunitaria del Filipino Women's Council. Acceso el 15 de febrero de 2017. http:// docplayer.it/11854288-lo-noi-e-loro-realta-e-illusioni-delle-collaboratricifamiliari-filippine-un-progetto-di-ricerca comunitaria-del-filipino-women-scouncil.html.

Bea, Giuseppe. 2012. "L'imprenditoria degli immigrati asiatici in Italia come ponte per lo sviluppo, Sede Nazionale della Confederazione Nazionale dell'Artigianato e della Piccola e Media Impresa e del Patronato EpasaCna». En Fourth Intercontinental Study Tour on Immigration del gruppo di ricercatori di Caritas y Migrantes in collaborazione con Scalabrini Migration Center di Manila. Actas del Congreso "Asia-Italia: scenari migratori», Centro Studi e Ricerche Idos, 14-20 de enero, Manila. Acceso el 2 de marzo de 2018. http://www.dossierimmigrazione.it/docnews/file/2012_ Asia_Italia_Relazioni\%20brevi.pdf.

Beacco, Jean Claude, Little, David y Chris Hedges. 2005. «L'integrazione linguistica dei migranti adulti. Guida per l'elaborazione di strategie e la loro attuazione». Italiano Lingua Due 6, n. 2: 1-37.

Bermejo, Rut. 2008. «Idioma e inmigración: un análisis de la evolución de los requisitos idiomáticos en las políticas de inmigración». Documento de Trabajo 17, 04.

Biondi Dal Monte, Francesca. 2017. «La lingua come strumento di integrazione scolastica delle nuove minoranze tra prospettive internazionali e diritto interno». Osservatoriosullefonti. it, 1-29. 
Broeder, Peter y Laura Mijares. 2003. Plurilingüismo en Madrid. Las lenguas de los alumnos de origen inmigrante en primaria. Madrid: CIDE.

Cama, Maria. 2017. «El conocimiento del italiano: requisito para obtener el Permiso de Residencia de larga duración CE. El caso de la comunidad filipina de Mesina (Italia)». Revista Internacional de Estudios Migratorios 7, n. 3: 303-327.

Cardullo, Stefania. 2010. «Le nuove generazioni di immigrati: i ragazzi filippini a Messina». Quaderni di Intercultura, 17: 1-18.

Carpani, Daniela, Laura Sanfelici y Rosana Ariolfo. 2011. "La lengua como signo identificador: los desafíos educativos en el contexto multicultural genovés». Lengua y migración 3, n. 1: 53-72.

Colaiacomo, Alberto. 2012. "Le rimesse e il loro utilizzo sociale e produttivo, Dossier Statistico Immigrazione Caritas/Migrantes». En Fourth Intercontinental Study Tour on Immigration del gruppo di ricercatori di Caritas y Migrantes in collaborazione con Scalabrini Migration Center di Manila. Actas del Congreso «Asia-Italia: scenari migratori», Centro Studi e Ricerche Idos, 14-20 de enero, Manila. Acceso el 2 de marzo de 2018. http://www.dossierimmigrazione.it/ docnews/file/2012_Asia_Italia_Relazioni\%20brevi.pdf

Comune di Bologna. 2010. Cittadini stranieri a Bologna. Schede tematiche sulle nazionalità più diffuse. 2. Filippine. Acceso el 1 de marzo de 2018. http://www.comune.bologna.it/iperbole/piancont/Stranieri/StudiStranieri/ Stranieri_aBo/2010/pdf/Filippine.pdf

Enríquez, Maura Cruz. 2015. «Migrantes cubanos en Montreal: una adaptación vista desde el habla». Revista Lengua y migración 7, n. 2: 29-48.

Favaro, Graziella y Mara Tognetti. 1991. Donne dal mondo. Strategie migratorie al femminile. Milano: Guerini Associati.

Fellini, Ivana, Raffaele Guetto y Emilio Reyneri. 2018. «Poor returns to origincountry education for non-Western immigrants in Italy: An analysis of occupational status on arrival and mobility». Social Inclusion 6, n. 3: 34-47.

Firpo, Elena y Laura Sanfelici. 2016. La visione eteroglossica del bilinguismo: spagnolo lingua d'origine e Italstudio. Modelli e prospettive tra gli Stati Uniti e I'Italia. Milano: LED Edizioni.

García Castaño, Francisco J., Rafael Pulido y Ángel Montes del Castillo. 1993. «La educación multicultural y el concepto de cultura. Una visión desde la antropología social y cultural». Revista de Educación 302: 83-110.

García Castaño, Francisco J., María Rubio José Fernández Echeverría. 2018. «Las trampas de la diversidad. Sobre la producción de diferencias en la escuela». Gazeta de Antropología 34: 61-86

Gutiérrez, Rodolfo, Javier Mato y María Miyar. 2010. «Lengua e inmigración: conocimiento del español e integración de los inmigrantes». Migraciones internacionales 85: 47-64.

Hamel, Rainer E. 1995a. «Presentación». Alteridades 5, n. 10: 3-9. Acceso el 10 de febrero de 2020. http://www.redalyc.org/articulo.oa?id=74711345001.

Hamel, Rainer E. 1995b. «Derechos lingüísticos como derechos humanos: debates y perspectivas». Alteridades 5, n. 10: 11-23. 
Hipperdinger, Yolanda. 2015. "Ethnic revival: actitudes, políticas y usos lingüísticos de los alemanes del Volga en Argentina». Revista Lengua y migración 7, n. 2: 7-27.

IDOS/Immigrazione Dossier Statistico. 2014. La collettività filippina in Italia. Acceso el 1 de marzo de 2018. http://www.dossierimmigrazione.it/ docnews/file/2014_La\%20collettivit\%C3\%A0\%20filippina_Scheda.pdf.

ISTAT. 2019. Stranieri residenti - Cittadinanza. Acceso el 10 de abril de 2020 en http://dati.istat.it/Index.aspx?DataSetCode=DCIS_POPSTRCIT1 .

Italialavoro. 2015. "Servizio di rafforzamento in bagno linguistico delle competenze linguistiche, culturali e didattiche di 12 docenti di lingua italiana della TESDA - Repubblica delle Filippine. Italia». Documento multicopiado.

Joppke, Christian. 2007. "Transformation of citizenship: status, rights, identity». Citizenship Studies 11, n. 1: 37-48.

Kloss, Heinz. 1977 The American Bilingual Tradition. Rowley: Newbury House.

Malfone, Carlotta. 2006. «Immigrazione al femminile. Modelli femminili, valori, identità». Pedagogia Interculturale, Sociale e della Cooperazione 1: 1-22.

Martín Rojo, Luisa. 2010. Constructing inequality in multilingual classrooms. Berlín: Walter de Gruyter.

Martín Rojo, Luisa y Laura Mijares. 2007. «'Sólo en español': Una reflexión sobre la norma monolingüe y la realidad multilingüe en los centros escolares». Revista de Educación 343: 93-112.

May, Steven. 2010. «Derechos lingüísticos come derechos humanos». Revista de Antropología Social 19: 131-159

Ministero del Lavoro e delle Politiche Sociali, (Direzione Generale dell'Immigrazione e delle Politiche di Integrazione). 2016. La Mobilità Internazionale del Lavoro. FILIPPINE. Scheda paese. Documento multicopiado.

Ministero del Lavoro e delle Politiche Sociali. (Direzione Generale dell'Immigrazione e delle Politiche di Integrazione). 2018. "La Comunità Filippina in Italia». Rapporto annuale sulla presenza dei migranti. Acceso el 5 de abril de 2020. http://www.integrazionemigranti.gov.it/Areetematiche/ PaesiComunitari-e associazioniMigranti/paesi/Pagine/Filippine.aspx

Morales, Julieta. (2017). "Derechos humanos y migraciones: Paradojas y reformulaciones en 2017». Ciencia Jurídica 11: 91-118.

Olmos, Antonia (2016). "Diversidad lingüístico-cultural e interculturalismo en la escuela andaluza: Un análisis de políticas educativas». Relieve 22, n. 2: 1-20. doi: http://dx.doi.org/10.7203/relieve.22.2.683

Pozzi, Sonia. 2014. "Trasmissione delle lingue, integrazione e identità nelle famiglie immigrate». En Lingue migranti e nuovi paesaggi, editado por Maria Calvi Bajini I. Vittoria y Milin Bonomi, vol. 1, 37-50. Milano: LED Edizioni Universitarie.

Rubio, Raquel (2015). «Investigaciones sobre la enseñanza del español y su cultura en contextos de inmigración». Lengua y migración/Language and Migration 7, n. 2: 109-115

Sobrero, Alberto y Annarita Miglietta. 2011. "Per un approccio varietistico all'insegnamento dell'italiano a stranieri». Italiano LinguaDue 3, n. 1: 233-260. 
TESDA (Technical Education and Skill Development Authority). 2015. Training of Trainers on Italian Language and Culture. República de Filipinas: Manila. Documento multicopiado.

Tognetti, Mara. 2004. «Lavoro e immigrazione femminile in Italia: una realtà in mutamento». En Immigrazione in Europa. Strategie di inclusioneesclusione, editado por Marcella Delle Donne y Umberto Melotti, 153-182. Roma: Ediesse Editore.

Vila, Ignasi. 1999. «Inmigración, educación y lengua propia». En La inmigración extranjera en España. Los retos educativos, editado por Eliseo Aja, 145166. Barcelona: Fundación La Caixa. 


\section{Copyright}

Deusto Journal of Human Rights / Revista Deusto de Derechos Humanos is an Open Access journal; which means that it is free for full and immediate access, reading, search, download, distribution, and reuse in any medium only for non-commercial purposes and in accordance with any applicable copyright legislation, without prior permission from the copyright holder (University of Deusto) or the author; provided the original work and publication source are properly cited (Issue number, year, pages and DOI if applicable) and any changes to the original are clearly indicated. Any other use of its content in any medium or format, now known or developed in the future, requires prior written permission of the copyright holder.

\section{Derechos de autoría}

Deusto Journal of Human Rights / Revista Deusto de Derechos Humanos es una revista de Acceso Abierto; lo que significa que es de libre acceso en su integridad inmediatamente después de la publicación de cada número. Se permite su lectura, la búsqueda, descarga, distribución y reutilización en cualquier tipo de soporte sólo para fines no comerciales y según lo previsto por la ley; sin la previa autorización de la Editorial (Universidad de Deusto) o la persona autora, siempre que la obra original sea debidamente citada (número, año, páginas y DOI si procede) y cualquier cambio en el original esté claramente indicado. Cualquier otro uso de su contenido en cualquier medio o formato, ahora conocido o desarrollado en el futuro, requiere el permiso previo por escrito de la persona titular de los derechos de autoría. 